\begin{tabular}{|c|l|}
\hline Title & Nuclear surface diffuseness revealed in nucleon-nucleus diffraction \\
\hline Author(s) & Hatakeyama, Shinya; Horiuchi, Wataru; Kohama, A. \\
\hline Citation & $\begin{array}{l}\text { Physical Review C, 97(5), 054607 } \\
\text { https://doi.org/10.1103/PhysRevC.97.054607 }\end{array}$ \\
\hline Issue Date & 2018-05-18 \\
\hline Doc URL & http://hdl.handle.net/2115/13556 \\
\hline Rights & @2018A merican Physical Society \\
\hline Type & article \\
\hline File Information & PhysRevC.97.054607.pdf \\
\hline
\end{tabular}

Instructions for use 


\title{
Nuclear surface diffuseness revealed in nucleon-nucleus diffraction
}

\author{
S. Hatakeyama, ${ }^{1}$ W. Horiuchi, ${ }^{1}$ and A. Kohama ${ }^{2}$ \\ ${ }^{1}$ Department of Physics, Hokkaido University, Sapporo 060-0810, Japan \\ ${ }^{2}$ RIKEN Nishina Center, RIKEN, Wako-shi, Saitama 351-0198, Japan
}

(Received 22 February 2018; published 18 May 2018)

\begin{abstract}
The nuclear surface provides useful information on nuclear radius, nuclear structure, as well as properties of nuclear matter. We discuss the relationship between the nuclear surface diffuseness and elastic scattering differential cross section at the first diffraction peak of high-energy nucleon-nucleus scattering as an efficient tool in order to extract the nuclear surface information from limited experimental data involving short-lived unstable nuclei. The high-energy reaction is described by a reliable microscopic reaction theory, the Glauber model. Extending the idea of the black sphere model, we find one-to-one correspondence between the nuclear bulk structure information and proton-nucleus elastic scattering diffraction peak. This implies that we can extract both the nuclear radius and diffuseness simultaneously, using the position of the first diffraction peak and its magnitude of the elastic scattering differential cross section. We confirm the reliability of this approach by using realistic density distributions obtained by a mean-field model.
\end{abstract}

DOI: 10.1103/PhysRevC.97.054607

\section{INTRODUCTION}

A nucleus is composed of protons and neutrons interacting via nuclear force. They make a self-consistent mean field that results in forming the nuclear shell structure. A systematic analysis of nuclear charge radii via the electron-elastic scattering have revealed that nuclei have saturated internal density and a relatively sharp surface that defines a nuclear radius [1]. Advances in the radioactive ion beam facilities have made it possible to study properties of short-lived unstable nuclei. From such facilities, the exotic structure of neutron-rich unstable nuclei was reported, which has never been observed in stable nuclei, e.g., halo [2] and developed skin [3] structure.

Such exotic structure is dominated by nuclear dynamics at around the nuclear surface. For example, nuclear deformation plays a crucial role in enlarging the nuclear radius, because it drastically changes the density profile at around the nuclear surface, and has actually been confirmed by the systematic analyses of the total reaction cross sections on a carbon target [4-10]. Also, it is found that excitations of the outermost single-particle neutron orbits play an essential role to determine the low-lying electric-dipole strengths of neutron-rich isotopes [11-14].

Since the density profile at around the nuclear surface is a rich source of the nuclear structure information, a systematic investigation of the nuclear surface density distributions must be worth studying. However, the neutron density distribution is difficult to probe by the traditional electron scattering. Alternatively, the proton-elastic scattering is suitable for that purpose [15]. Recent precise measurements up to large scattering angles were successful in extracting the neutron density distributions of $\mathrm{Sn}$ and $\mathrm{Pb}$ isotopes with the help of known proton density distributions [16,17]. To apply it for unstable nuclei, such measurement in the inverse kinematics is useful but it is not easy to obtain the cross sections at large scattering angles because most of incident particles are scattered in the forward angles at high incident energies. Since precise experimental cross sections are limited to small scattering angles, it is convenient to know what information we can obtain from limited cross-section data.

In this paper, we perform a numerical experiment systematically using theoretically obtained nucleon-nucleus scattering cross sections focusing on the reactions of small scattering angles up to a few diffraction peaks to see to what extent the information on the nuclear surface can be obtained. For this purpose, we start with an idea of a simple black sphere (BS) picture, which assumes a nucleus is a completely absorptive object at a sharp-cut square-well radius. The model is mathematically equivalent to the Fraunhofer diffraction model $[18,19]$, which offers one-to-one correspondence between the nuclear radius and the diffraction peak position. Though it is not perfect, the idea can be a zeroth-order approximation of the proton-nucleus scattering, remarking the fact that the BS model explains fairly well a systematic trend of the proton-nucleus total reaction cross sections, which was originally pointed out by Kohama et al. [20-22].

In reality, since the total nucleon-nucleon cross section is not large enough at medium and high incident energies, the proton-nucleus scattering is not completely absorptive at around the nuclear surface where the nuclear density is not well saturated. A simple model approach based on the BS picture and the proton optical depth shows that the effect of the surface diffuseness plays an essential role to determine the incident energy dependence of the total reaction cross sections of the proton-nucleus scattering [22,23]. Here we discuss the role of the nuclear transparency due to the surface diffuseness, which is not explicitly taken into account in the BS model by comparing it with a microscopic high-energy reaction theory, the Glauber model [19]. To understand the significance of its 
good reproducibility of the data using such a phenomenology is another purpose of this paper.

This paper is organized as follows. In the next section, we briefly explain calculations of the elastic scattering differential cross section of the high-energy nucleon-nucleus scattering in the Glauber model. In Sec. III A, we demonstrate how the elastic scattering differential cross section reflects the density profile at the nuclear surface. A systematic analysis is performed by using a two-parameter Fermi $(2 \mathrm{pF})$ distribution as the density profile, which clearly defines the nuclear diffuseness. We find one-to-one correspondence between the nuclear diffuseness and the magnitude of the cross section at the first peak position. To quantify the sensitivity to the density profile at around the surface region, we investigate in Sec. III B the spatial distributions of the scattering amplitude at the first and second peak positions of the elastic scattering differential cross sections. In Sec. IIIC, we propose a simple way to extract both the nuclear radius and diffuseness information from the elastic scattering differential cross sections for future application to short-lived unstable nuclei. We extract the information on the surface diffuseness of density distributions obtained via a microscopic mean-field approach. By assuming the $2 \mathrm{pF}$ density distributions, the unknown diffuseness and nuclear radius are uniquely determined in such a way that the first peak position and its magnitude of the elastic scattering differential cross section are reproduced simultaneously. It will be convenient to know the first peak position before measurement from other observables in which we show, in Sec. IIID, a relationship between the first peak position and the total reaction cross section with the help of the BS model. The possibility of extracting proton and neutron diffuseness separately is discussed in Sec. III E. Conclusions are presented in Sec. IV.

\section{ELASTIC SCATTERING DIFFERENTIAL CROSS SECTION IN THE GLAUBER MODEL}

The Glauber model is a microscopic theory, which is widely used to study high-energy nucleus-nucleus collisions [19]. With the help of the adiabatic and eikonal approximations, the final-state scattering wave function is greatly simplified as

$$
\left|\Phi_{f}\right\rangle=e^{i \chi}\left|\Phi_{i}\right\rangle
$$

where $e^{i \chi}$ is the so-called phase-shift function, which includes all information of the high-energy nuclear collision. The elastic scattering differential cross section can be calculated by

$$
\frac{d \sigma}{d \Omega}(\theta)=|F(\theta)|^{2}
$$

with the elastic scattering amplitude

$$
F(\theta)=\frac{i K}{2 \pi} \int e^{-i \boldsymbol{q} \cdot \boldsymbol{b}}\left(1-e^{i \chi(\boldsymbol{b})}\right) d \boldsymbol{b},
$$

where $K$ is the wave number in the relativistic kinematics, $\boldsymbol{q}$ the momentum transfer vector, and $\boldsymbol{b}$ is the impact parameter vector perpendicular to the beam direction $(z)$, and thus $\boldsymbol{q} \cdot \boldsymbol{b}=2 K b \sin \frac{\theta}{2}$. Evaluation of $e^{i \chi(\boldsymbol{b})}$ is in general difficult because it involves multiple integration [19]. Though it could be possible to perform the integration by using a Monte Carlo technique $[24,25]$ or a factorization procedure by using a Slater determinant wave function [26-29], we, however, employ the optical-limit approximation (OLA) for the sake of simplicity. The phase-shift function of the OLA is given as the leading order of the cumulant expansion of the full phase-shift function $[19,30]$

$$
i \chi(\boldsymbol{b}) \simeq-\sum_{N=p, n} \int \rho_{N}(\boldsymbol{r}) \Gamma_{p N}(\boldsymbol{b}-\boldsymbol{s}) d \boldsymbol{r},
$$

where $\boldsymbol{r}=(\boldsymbol{s}, z)$ with $s$ being a two-dimensional vector perpendicular to $z$. Inputs to the theory are density distributions $\rho_{N}(\boldsymbol{r})$ of proton $(N=p)$ and neutron $(N=n)$, and the proton-nucleon profile function $\Gamma_{p N}$. As exemplified in Refs. [24-26,28,29], the OLA works well for many cases of nucleon-nucleus scattering. The multiple scattering effect would be neglected and even becomes smaller for systems involving medium to heavy nuclei as was shown in Refs. [28,29].

The nucleon-nucleon profile function at incident energy per nucleon $E$ is usually parametrized as [31]

$$
\Gamma_{N N}(\boldsymbol{b}, E)=\frac{1-i \alpha_{N N}(E)}{4 \pi \beta_{N N}(E)} \sigma_{N N}^{\mathrm{tot}}(E) \exp \left[-\frac{\boldsymbol{b}^{2}}{2 \beta_{N N}(E)}\right],
$$

where $\alpha_{N N}$ is the ratio of the real and imaginary parts of the scattering amplitude at the forward angle, and $\beta_{N N}$ is a slope parameter. For the sake of simplicity, we first use averaged $N N$ profile function given in Ref. [32] for most of discussions made in this paper. We can safely use the profile function, say $E \gtrsim 300 \mathrm{MeV}$, where the difference between $p n$ and $p p$ cross sections are not significant. The validity of adopting the averaged $N N$ profile function here is discussed in Appendix. We distinctively use the $p n$ and $p p$ profile functions [33] when more realistic cases are considered in Sec. IIIE. The spin-orbit term is not explicitly included in the profile function [33]. As long as we are focusing on the analysis of the elastic scattering differential cross sections at the forward angles, this effect is small at the peak positions [20], whereas the cross sections at the diffraction minima are significantly influenced by the spin-orbit interaction [34]. In fact, as shown in Ref. [35], the elastic scattering differential cross-sections data at the forward angles are fairly well reproduced using the profile function (5) without the spin-orbit term. The elastic and inelastic Coulomb contributions are ignored since the effects are negligible in the proton-nucleus scattering [35].

\section{RESULTS}

In this section, we show the results of the analyses explained in the previous section.

\section{A. Elastic scattering differential cross section and nuclear surface distribution}

Here we discuss how much information the elastic scattering cross sections of the first diffraction peak has on the nuclear surface. In the present work, we are interested in medium to heavy nuclei whose central densities are well saturated. It would be reasonable to assume a two-parameter Fermi $(2 \mathrm{pF})$ 

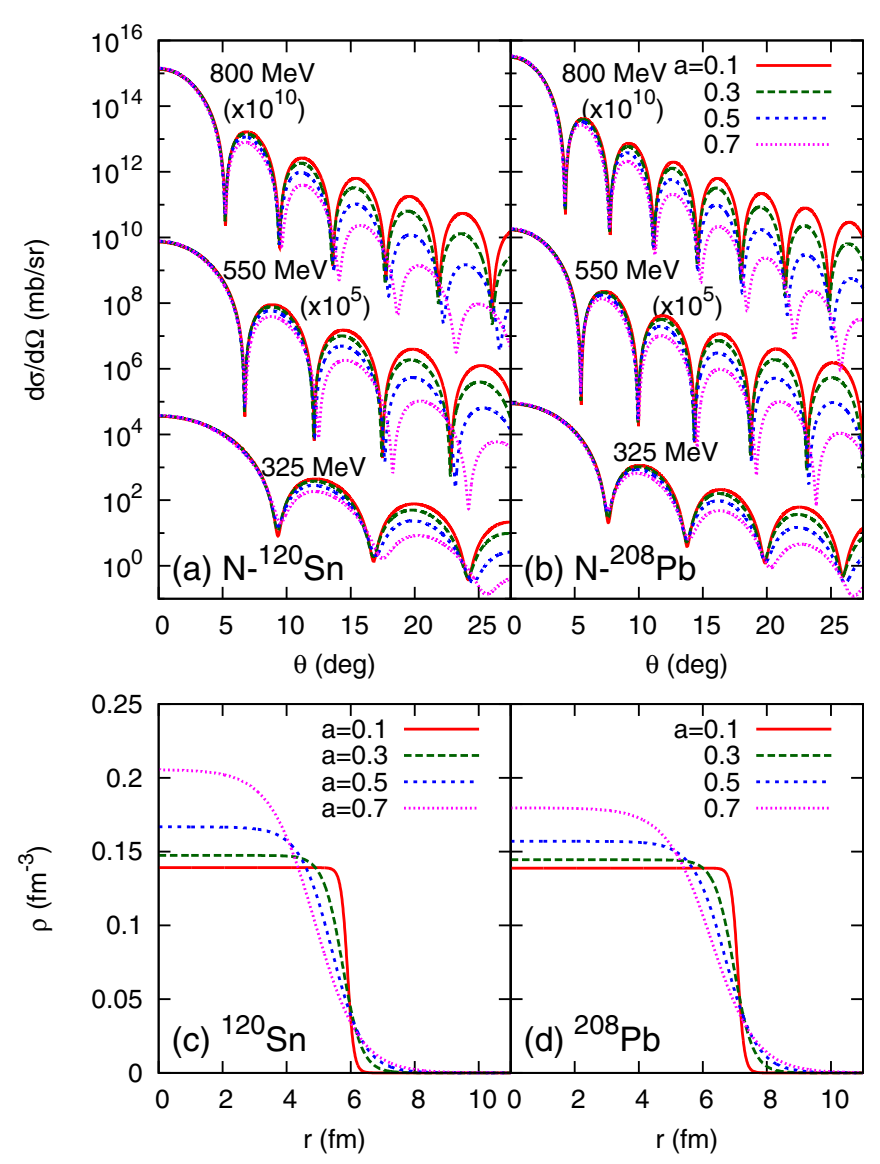

FIG. 1. Elastic scattering differential cross sections of (a) $N-{ }^{120} \mathrm{Sn}$ and (b) $N_{-}{ }^{208} \mathrm{~Pb}$ systems calculated with $2 \mathrm{pF}$ density distributions at 325,550 , and $800 \mathrm{MeV}$. The cross sections are multiplied by $10^{5}$ and $10^{10}$ for those at 550 and $800 \mathrm{MeV}$, respectively. The bottom panels plot the corresponding $2 \mathrm{pF}$ density distributions of (c) ${ }^{120} \mathrm{Sn}$ and (d) ${ }^{208} \mathrm{~Pb}$, respectively, with various diffuseness parameter $a$. All density distributions give the same root-mean-square radius.

function as an approximate nuclear density distribution:

$$
\rho(r)=\frac{\rho_{0}}{1+\exp \left(\frac{r-R}{a}\right)},
$$

where $\rho_{0}, R$, and $a$ are the central density, radius, and diffuseness parameters, respectively. For given $R$ and $a$, $\rho_{0}$ is uniquely determined by the normalization condition: $4 \pi \int_{0}^{\infty} \rho(r) r^{2} d r=A$, where $A$ is the mass number of a nucleus. The root-mean-square (rms) matter radius can be calculated by

$$
r_{m}=\sqrt{\left\langle r^{2}\right\rangle}=\sqrt{\frac{4 \pi}{A} \int_{0}^{\infty} d r r^{4} \rho(r)} .
$$

We note that the limit $a \rightarrow 0$ in Eq. (6) results in a sharp-cut square-well density distribution with a radius $R=\sqrt{\frac{5}{3}} r_{m}$.

We perform the Glauber model calculation with the $2 \mathrm{pF}$ density distribution of Eq. (6). The rms radius of the $2 \mathrm{pF}$ density distribution is set to follow the empirical rms radius $r_{m}=\sqrt{\frac{3}{5}} 1.2 A^{1 / 3}[36]$ as a convenient choice so that the radius parameter $R$ is determined for each given $a$. Note that the

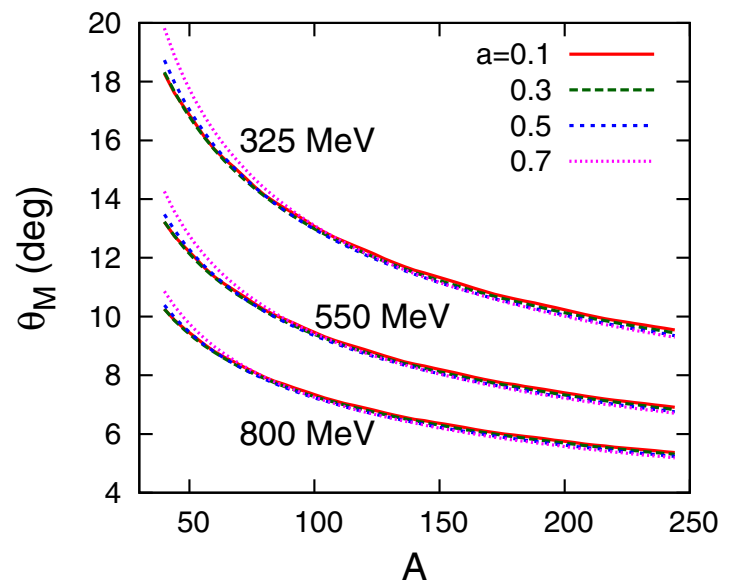

FIG. 2. Scattering angles at the first peak positions of the elastic scattering differential cross sections incident at 325, 550, and $800 \mathrm{MeV}$ as a function of mass number.

resultant $R$ is in general different from the radius obtained by the sharp-cut square-well density distribution, $\sqrt{\frac{5}{3}} r_{m}$.

\section{Nuclear radius $\leftrightarrow$ First peak position of the elastic scattering differential cross section}

First, we discuss the relation between the nuclear radius and the scattering angle at the first peak position of the elastic scattering differential cross section. Figure 1 plots the elastic scattering differential cross sections of $N-{ }^{120} \mathrm{Sn}$ and $N-{ }^{208} \mathrm{~Pb}$ systems incident at 325,550 , and $800 \mathrm{MeV}$ with various diffuseness parameter, $a$. Corresponding $2 \mathrm{pF}$ density distributions are also plotted in the bottom panels of Fig. 1. Focusing on the first diffraction peak, all the cross sections are peaked at almost the same scattering angle for all incident energies under consideration. Since the BS model works well in the nucleon-nucleus scattering, the one-to-one correspondence between the peak position and the nuclear radius can be naturally understood, remarking that all the $2 \mathrm{pF}$ density distributions give the same rms radius.

For more quantitative discussions, we display, in Fig. 2, the scattering angles at the first peak position, $\theta_{M}$, as a function of mass number. Incident energies of 325, 550, and $800 \mathrm{MeV}$ are chosen. We again confirm that the first peak positions do not depend on the diffuseness parameter of the $2 \mathrm{pF}$ density distribution. The peak position is determined mostly by the nuclear radius. For a small $A \lesssim 70$, the $\theta_{M}$ values show some dependence on $a$, especially with large $a=0.7 \mathrm{fm}$. Since we assume the $2 \mathrm{pF}$ distribution, in the case of small $A$, i.e., small $R$, large diffuseness parameter substantially affects the density profile at small distances, which is a large increase of the central density as already seen in the bottom panels of Fig. 1 .

\section{Nuclear diffuseness $\leftrightarrow$ Magnitude of elastic scattering differential cross section at the first peak position}

Second, we discuss the relation between the nuclear diffuseness and the magnitude of the elastic scattering differential cross section at the first peak position. In Fig. 1, it is interesting to note that the elastic scattering differential cross sections at 


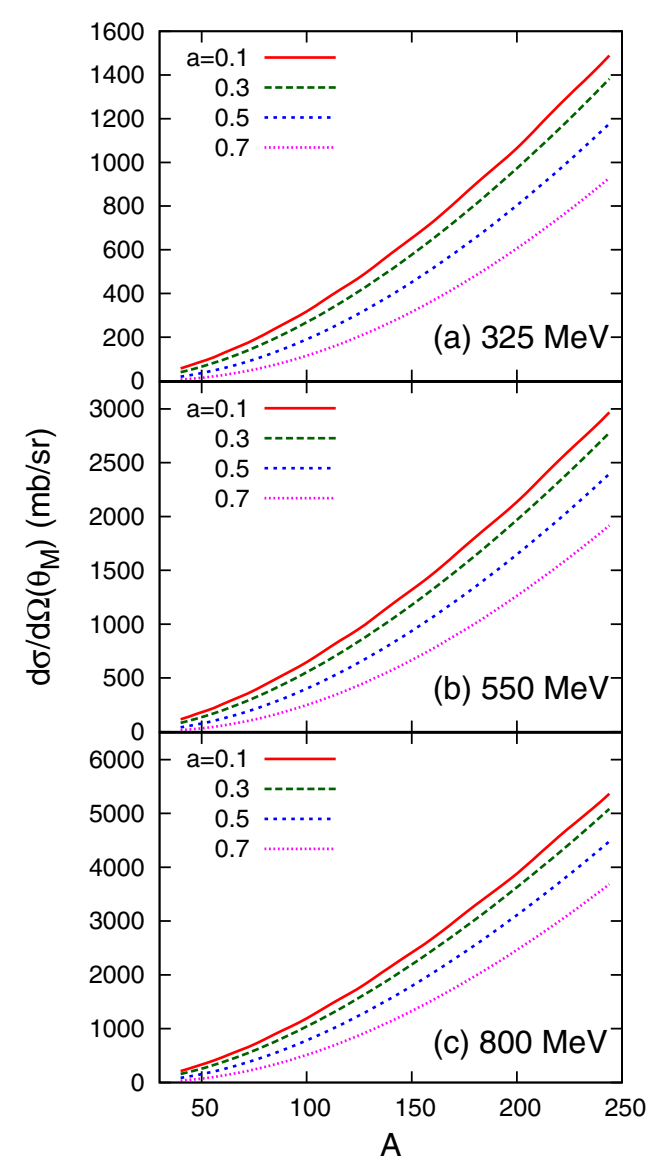

FIG. 3. Elastic scattering differential cross sections at the first peak position incident at (a) 325 , (b) 550 , and (c) $800 \mathrm{MeV}$ as a function of mass number.

the first peak position are mostly determined by $a$. The authors of Ref. [37] pointed out a relation between the nuclear surface diffuseness and the elastic scattering differential cross section, in which the cross section at the first peak position is enhanced with smaller nuclear diffuseness. The calculated cross sections actually show a larger value at the first peak position with smaller $a$.

Figure 3 plots the magnitude of the elastic scattering differential cross section at the scattering angle of the first peak position $\theta_{M}$ as a function of mass number. Incident energies of 325,550 , and $800 \mathrm{MeV}$ are chosen. The cross section significantly decreases with increasing $a$, which would easily be distinguished by measurement. Though the sensitivity to $a$ becomes a little bit less at $800 \mathrm{MeV}$, higher incident energy gives larger cross sections. We find that for a given $A$, i.e., rms radius, the cross sections with different $a$ do not intersect each other for all the incident energies, indicating that the $R$ and $a$ parameters of the $2 \mathrm{pF}$ density distribution can uniquely be determined if the elastic scattering differential cross section is measured at the first peak position.

\section{B. Scattering amplitude at the first and second peak positions}

We have seen so far that the nuclear diffuseness information can be extracted from the elastic scattering differential cross

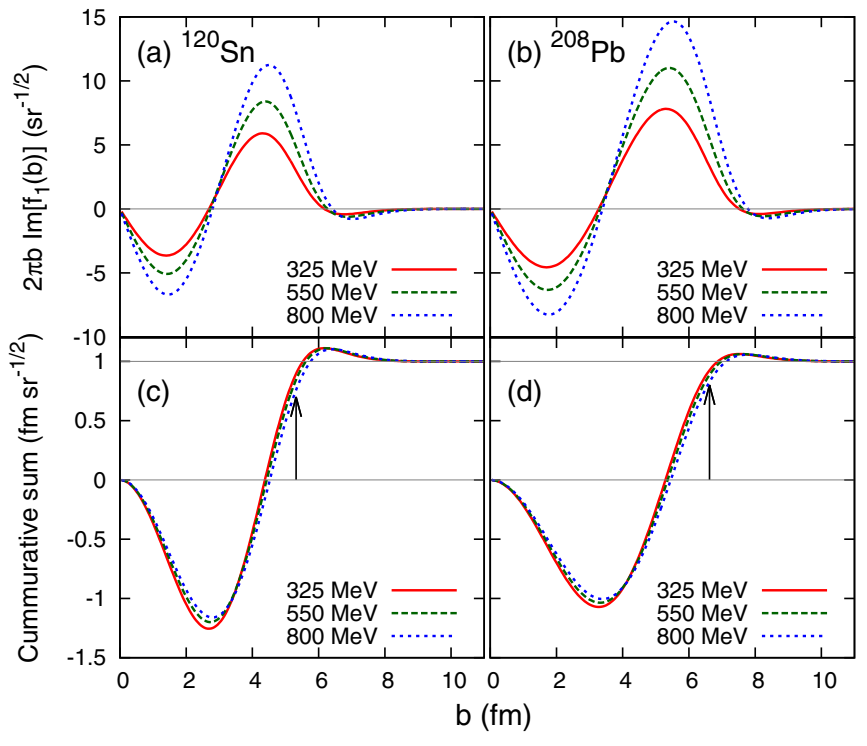

FIG. 4. (Top) Imaginary part of the spatial distribution of the scattering amplitude, and (bottom) its cumulative sum at the first peak of the elastic scattering differential cross section of (a), (c) ${ }^{120} \mathrm{Sn}$ and (b), (d) ${ }^{208} \mathrm{~Pb}$, as a function of impact parameter $b$, incident at 325,550 , and $800 \mathrm{MeV}$. An arrow indicates the half-density radius of (c) ${ }^{120} \mathrm{Sn}$ and (d) ${ }^{208} \mathrm{~Pb}$, respectively. See text for details.

section at the first peak position. In this section, we discuss what the incident nucleon actually probes. To answer this, we calculate the scattering amplitude of the differential cross section at the first peak position $\theta_{M}$ as a function of the impact parameter $b=|\boldsymbol{b}|$

$$
f_{1}(\boldsymbol{b})=\frac{i K}{2 \pi} e^{-i \boldsymbol{q}_{M} \cdot \boldsymbol{b}}\left(1-e^{i \chi(\boldsymbol{b})}\right),
$$

where $\boldsymbol{q}_{M} \cdot \boldsymbol{b}=2 K b \sin \frac{\theta_{M}}{2}$, and the relation to the scattering amplitude at the first peak position

$$
F\left(\theta_{M}\right)=\int f_{1}(\boldsymbol{b}) d \boldsymbol{b} .
$$

Figure 4 plots the imaginary part of the spatial distribution of the scattering amplitude of Eq. (8) for ${ }^{120} \mathrm{Sn}$ and ${ }^{208} \mathrm{~Pb}$ and its cumulative sum defined by $2 \pi b \int_{0}^{b} \operatorname{Im} f_{1}\left(b^{\prime}\right) d b^{\prime} / \operatorname{Im} F\left(\theta_{M}\right)$, at various incident energies as a function of impact parameter $b$. The real part is not shown because it is small. The diffuseness parameters are set commonly to an empirical value $a=0.54 \mathrm{fm}$ [36]. The half-density radius $\rho\left(R_{h}\right)=\rho_{0} / 2$ for each nuclide is indicated by an arrow. All curves exhibit positive and negative peaks inside the nuclear half-radius. The cumulative sum of ${ }^{120} \mathrm{Sn}\left({ }^{208} \mathrm{~Pb}\right)$ indicates that the amplitude up to $\sim 4.5 \mathrm{fm}(\sim 5.5 \mathrm{fm})$ does not contribute to the integrated scattering amplitude as they are canceled out through the integration over $b$. As a result, only the scattering amplitude at around $R_{h}$ is contributed to the cross sections in such a special kinematic condition. The nucleon-nucleus cross section at the first peak position can be a useful observable to extract the density profiles at around the half-density radius.

We comment on what information can be obtained in the higher-order diffraction peak. We see, in Fig. 1, that peak positions of the higher-order diffraction are shifted to 


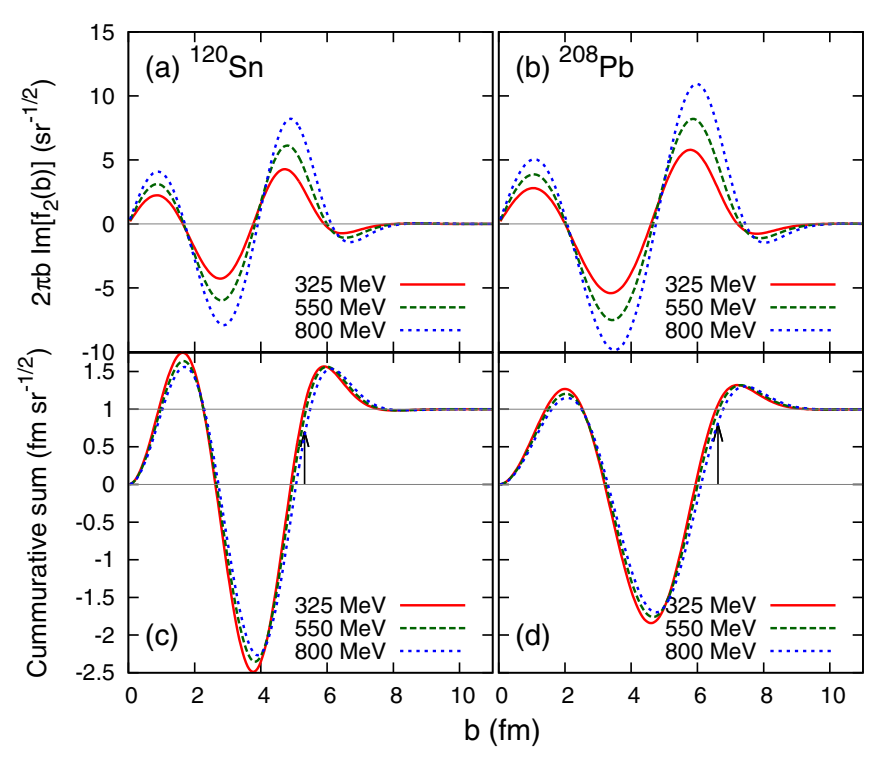

FIG. 5. Same as Fig. 4 but at the second peak of the elastic scattering differential cross section.

larger scattering angles with increasing $a$, implying different sensitivity to the nuclear density profile. Figure 5 plots the same quantity as Eq. (8) but at the second peak position, $f_{2}(\boldsymbol{b})$. The spatial distribution allows one more node and varies more rapidly with increasing $b$. The cumulative sum also oscillates and shows some contribution, reaching at the tail region, which is a bit distant from the half-density radius. The cross section at the second peak would have some other information on the density profile than that at the first peak. Further investigation would be interesting since it is useful to extract a higher order of the density profile beyond the half-density radius, which characterizes weakly bound systems, e.g., halo nuclei, but it is beyond the scope of this paper.

\section{Extraction of nuclear radius and diffuseness}

Thus far, we have discussed that the nuclear radius and diffuseness information is embedded in the first peak position and its magnitude of the elastic scattering cross section. In this section, we demonstrate how we can extract the nuclear diffuseness as nuclear structure information when the nucleon- nucleus elastic scattering differential cross sections are given. For this purpose, we employ general proton and neutron density distributions obtained by a microscopic mean-field model as inputs to the Glauber model.

We take the density distributions of $\mathrm{Ca}, \mathrm{Ni}, \mathrm{Zr}, \mathrm{Sn}, \mathrm{Yb}$, and $\mathrm{Pb}$ isotopes obtained by the Skyrme-Hartree-Fock $(\mathrm{HF})+$ BCS method [38] used in Refs. [35,39] (one can also take them from the theoretical database [40]). The calculation was performed self-consistently in a three-dimensional Cartesian mesh, in which any nuclear deformation can be taken into account. The density distribution in the laboratory frame is obtained by taking an average on the angles as in Ref. [8]. We remark that the theoretical justification of this treatment was made in Ref. [7]. The Skyrme-type effective interaction (SkM* [41]) with a monopole-type pairing interaction is employed as detailed in Refs. [13,42]. The SkM* parameter set is superior to describe the nuclear deformation. For example, kink behavior due to the nuclear deformation in the total reaction cross sections of neutron-rich $\mathrm{Ne}$ and $\mathrm{Mg}$ isotopes are reproduced very well with the help of the Glauber model $[8,43]$.

To deduce the diffuseness of the realistic density distribution through reaction data, we calculate the elastic scattering differential cross sections with the $2 \mathrm{pF}$ density distribution of Eq. (6). Regarding that those calculated cross sections with the HF+BCS density distributions are experimental data, we determine the $R$ and $a$ in the $2 \mathrm{pF}$ density distribution in such a way that the calculated elastic scattering differential cross section matches the first peak position as well as its magnitude of the elastic scattering differential cross section obtained by the numerical experiment. To assure the accuracy of the extracted $R$ and $a$, we confirm that our cross-section calculations are converged within four digits.

\section{Uncertainties of the extraction}

Following the procedure mentioned above, we determine the parameters in the $2 \mathrm{pF}$ density distributions for each isotope and each incident energy. In this section, we evaluate the robustness of this analysis.

Figure 6 plots the relative deviations of the rms radius obtained by the HF+BCS density, $r_{m}(\mathrm{HF})$, and that extracted from this analysis, $r_{m}(2 \mathrm{pF})$ for $\mathrm{Ca}, \mathrm{Ni}, \mathrm{Zr}, \mathrm{Sn}, \mathrm{Yb}$, and $\mathrm{Pb}$ isotopes. These results indicate the difference between realistic

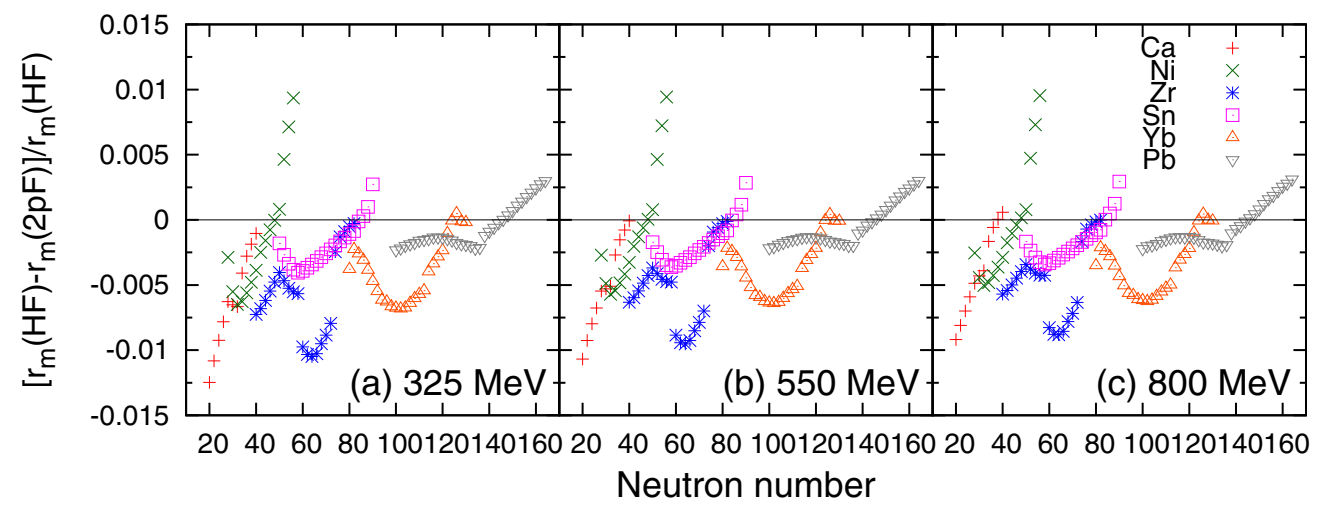

FIG. 6. Relative deviations of the rms radius obtained by the HF+BCS method and the deduced rms radii incident at (a) 325, (b) 550, and (c) $800 \mathrm{MeV}$. 


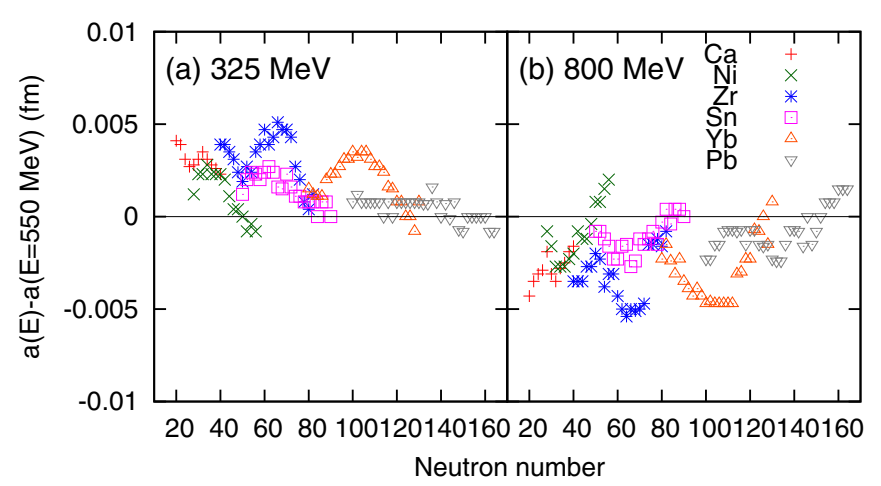

FIG. 7. Deviations of the nuclear diffuseness deduced at (a) 325 and (b) $800 \mathrm{MeV}$ from that at $550 \mathrm{MeV}$. See text for details.

$\mathrm{HF}+\mathrm{BCS}$ and simple $2 \mathrm{pF}$ density distributions, which will be commented later in this section and Sec. IIIC2. Here we choose three incident energies (a) 325, (b) 550, and (c) $800 \mathrm{MeV}$. The deviations are typically less than $1 \%$ for all the incident energies. The extracted rms radius agrees very well with the correct rms radius and is successfully obtained from this analysis. The $2 \mathrm{pF}$ density distribution can be a reasonable approximation to simulate the realistic density distributions of medium- to heavy-mass nuclei.

For the extraction of the diffuseness parameter $a$, we display in Fig. 7 the deviations of the diffuseness parameter $a$ at 325 and $800 \mathrm{MeV}$ from that at $550 \mathrm{MeV}$. The incidentenergy dependence is small at most by $\sim 0.005 \mathrm{fm}$. Though some systematic errors exist, which come from the difference between the realistic and $2 \mathrm{pF}$ density distributions, we can however determine, within this model approach, such an $a$ value in the accuracy of two digits. The $a$ value, which is extracted in this way, can be used as a measure of the surface diffuseness for the realistic density distribution.

Figure 8 compares the HF+BCS density with the $2 \mathrm{pF}$ distributions deduced from the first peak position and its

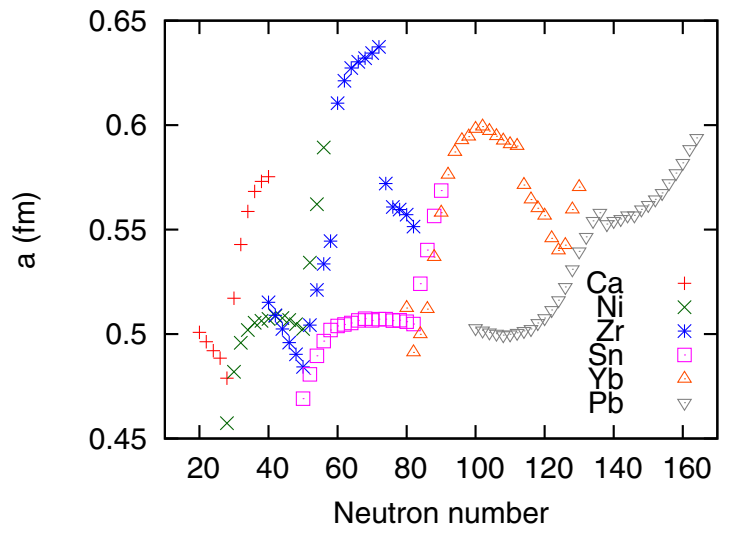

FIG. 9. Nuclear diffuseness deduced from the HF+BCS density distributions incident at $550 \mathrm{MeV}$.

magnitude of the elastic scattering differential cross section. The $2 \mathrm{pF}$ density distributions well simulate the HF one at around the nuclear surface at the three incident energies, $E_{1}=$ $325, E_{2}=550$, and $E_{3}=800 \mathrm{MeV}$. However, we see some deviations beyond $\sim 9 \mathrm{fm}$ for the neutron-rich $\mathrm{Zr}$ and $\mathrm{Sn}$ nuclei, which cannot be expressed by a simple $2 \mathrm{pF}$ distribution. This trend can also be seen in the behavior of the relative deviations of the rms radius plotted in Fig. 6. We also determine the $2 \mathrm{pF}$ density distribution by minimizing the root-mean-square deviation between the $2 \mathrm{pF}$ and $\mathrm{HF}+\mathrm{BCS}$ density distributions. As plotted in Fig. 8, the resultant $2 \mathrm{pF}$ density distributions are almost identical with those extracted from the first peak of the elastic scattering differential cross sections. The extracted $R$ and $a$ values can be robust structure information independently from the choice of the incident energy.

\section{Systematic trend of the nuclear diffuseness}

It is interesting to see the behavior of the nuclear diffuseness deduced from the HF+BCS density distributions. Figure 9

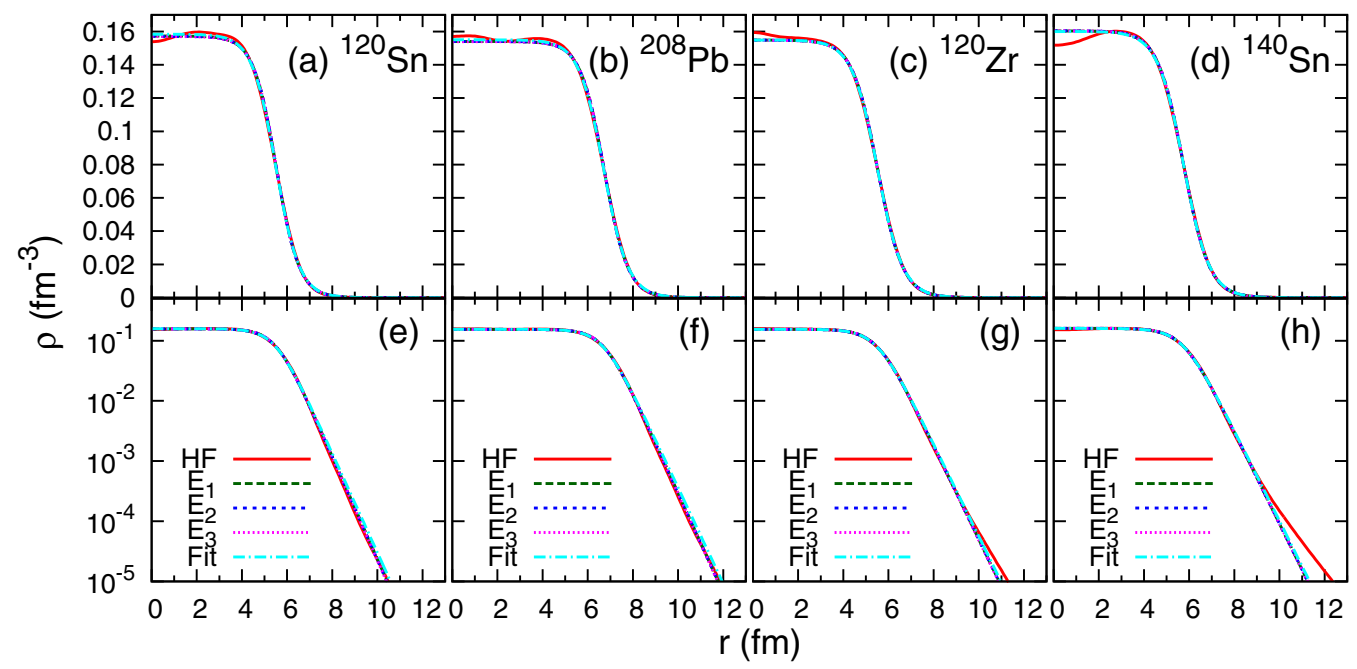

FIG. 8. Comparison of HF+BCS density and deduced $2 \mathrm{pF}$ density distributions of (a), (e) ${ }^{120} \mathrm{Sn}$; (b), (f) ${ }^{208} \mathrm{~Pb}$; (c), (g) ${ }^{120} \mathrm{Zr}$; and (d), (h) ${ }^{140} \mathrm{Sn}$ at different incident energies, $E_{1}=325, E_{2}=550$, and $E_{3}=800 \mathrm{MeV}$, drawn in (top) linear and (bottom) logarithmic scales. "Fit" denotes the $2 \mathrm{pF}$ density distribution deduced directly from the HF+BCS density. See text for details. 
displays the deduced $a$ values at $550 \mathrm{MeV}$. The $a$ values are scattered around $a \sim 0.5 \mathrm{fm}$, which is quite reasonable by pointing out the empirical value $\sim 0.54 \mathrm{fm}$ for stable nuclei [36]. As discussed in Ref. [39], the nuclear diffuseness is closely related to the width of the nuclear surface that determines the surface tension of the nuclear droplet. A systematic analysis of the surface widths clearly shows some exotic nuclear structure, such as nuclear deformation, and weakly bound orbits. We note that the diffuseness parameters or the surface widths extracted in this paper are those for the matter density distributions. The neutron number dependence is somewhat weaker than that of the neutron surface widths obtained in Ref. [39] because the proton surface widths are small and almost stay at a constant in the neutron-rich isotopes. Though it exhibits the weaker dependence on the neutron number than that of the neutron diffuseness, we can still see the structural information on the exotic neutron-rich isotopes. As expected, the $a$ values show local minima at the magic numbers. The $a$ values exhibit sudden rises at $N=50$ for $\mathrm{Ca}$ and $\mathrm{Ni}$ isotopes, and at $N=82$ for $\mathrm{Sn}$ isotopes, in which weakly bound neutron orbits play a role $[13,14]$. Large $a$ values in the open shell regions of $\mathrm{Zr}$ and $\mathrm{Yb}$ isotopes are due to the nuclear deformation, similarly to the cases of the $\mathrm{Ne}$ and $\mathrm{Mg}$ isotopes [5-9]. A systematic measurement of the elastic scattering differential cross sections covered up to the first diffraction peak will have of particular importance in order to reveal the evolution of the exotic structure of unstable nuclei.

It should be noted that this method may not be applicable to very weakly bound systems, such as halo nuclei, because the density profile deviates considerably from the $2 \mathrm{pF}$ assumption. To get higher resolution of the density profile, one may consider an analysis including higher-order diffraction peaks with more general density distribution other than the $2 \mathrm{pF}$ distribution.

\section{Black sphere estimate of the first peak position}

We have discussed that one can obtain the rms radius and nuclear diffuseness simultaneously from the first peak position and its magnitude of the elastic scattering differential cross section. It would be helpful to know the peak position before measurement of the elastic scattering differential cross sections. For this purpose, we investigate quantitative relation between the first peak position and the total reaction cross section using a concept of the strong absorption, i.e., the framework of the BS model [20].

If a nucleus is a completely absorptive object within a sharpcut nuclear radius $a_{\mathrm{BS}}$, the total reaction cross section reads exactly as

$$
\sigma_{\mathrm{BS}}=\pi a_{\mathrm{BS}}^{2}
$$

Note that the same thing holds for the total elastic cross section as well. The BS radius $a_{\mathrm{BS}}$ is obtained by the angle $\theta_{M}$ corresponding to the first diffraction peak as [20]

$$
a_{\mathrm{BS}}=\frac{5.1356 \cdots}{2 p \sin \left(\theta_{M} / 2\right)},
$$

where $p(=K)$ is the momentum between the two colliding particles.
As in Eq. (10), the total reaction cross section is directly related to the scattering angle of the first peak position, but, in reality, the total reaction cross section deviates from the one obtained from Eq. (10) due to the nuclear transparency, which comes from the surface diffuseness. In the Glauber model, the total reaction cross section is calculated by

$$
\sigma_{R}=\pi a_{R}^{2}=\int\left(1-\left|e^{i \chi(\boldsymbol{b})}\right|^{2}\right) d \boldsymbol{b},
$$

where we see similarity to Eq. (10) by introducing a reaction radius $a_{R}[35,44]$. In contrast, the $a_{\mathrm{BS}}$ is determined from the first peak position of the calculated elastic scattering differential cross section [20], using Eq. (11), and obtain $\sigma_{\mathrm{BS}}$ by the formula (10).

Figure 10 compares the total reaction cross sections incident at 325,550 , and $800 \mathrm{MeV}$ obtained by the Glauber calculation and the BS estimate using Eq. (10). The $2 \mathrm{pF}$ density distributions are employed. Since the BS model assumes the sharp-cut square-well nuclear surface and complete absorption in $r \leqslant a_{\mathrm{BS}}$, the deviation must include the information on the nuclear diffuseness or nuclear transparency at around the surface. If the nucleon-nucleus scattering is the ideal black sphere, all results will be on a $y=x$ line drawn in this figure. However, some deviation is found indicating the difference between the BS model and actual proton-nucleus scattering. Since more nucleons at around the nuclear surface contribute to the scattering process, the deviation becomes larger and larger with increasing nuclear size and diffuseness, which is typically by $\sim 5 \%$, at most by $\sim 10 \%$ at $\mathrm{Pb}$ isotopes in the ranges of the standard diffuseness parameters $a=0.5-0.6 \mathrm{fm}$.

We note that the energy dependence of the deviation cannot be explained simply by the magnitude of the total nucleon-nucleon cross section, but it comes from the difference of the nucleon-nucleon scattering processes or the profile functions in the present model, which is reflected in the nuclear transparency at around the surface. In fact, we confirm that the BS estimate gives the same cross section at any incident energy when the same profile function is used.

We have shown that the BS model explains more than $90 \%$ of the nucleon-nucleus scattering. Moreover, it is practically important to know how accurate we can obtain the first peak position if one converts the total reaction cross section to the scattering angle of the first peak position using the relation of Eq. (11). Figure 11 displays the difference between $\theta_{M}$ and $\theta_{R}$. The former can be obtained directly from the first peak position of the elastic scattering differential cross sections, and the latter can be calculated by converting the relation

$$
a_{R}=\sqrt{\frac{\sigma_{R}}{\pi}}=\frac{5.1356 \cdots}{2 p \sin \left(\theta_{R} / 2\right)},
$$

through Eq. (12). Again, we note that if the nucleon-nucleus scattering is completely absorptive, the difference must be zero. Despite the fact that the $\sigma_{\mathrm{BS}}$ slightly deviates from $\sigma_{R}$ as shown in Fig. 10, the differences of those scattering angles appear to be small, only within a few degrees. Therefore, Eq. (13) works well for the estimation of the first peak of the nucleon-nucleus diffraction, and thus the total reaction cross section can be complementary information to set up scattering angles to be covered by measurement. 


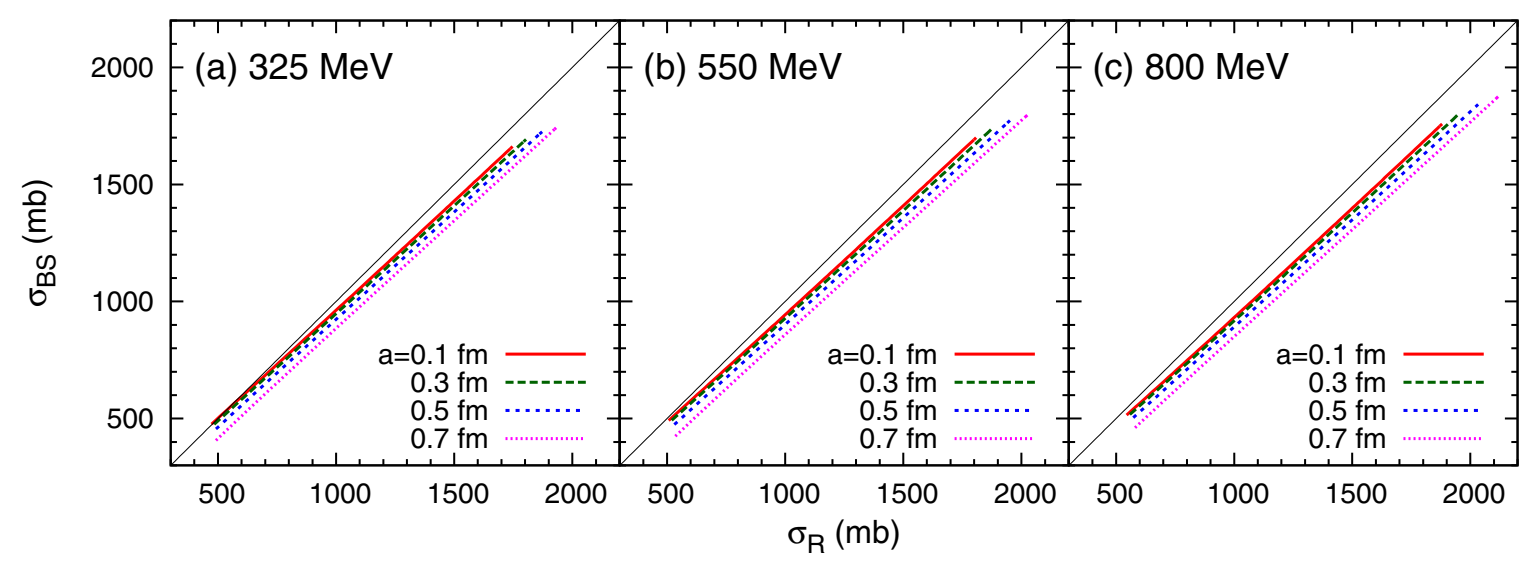

FIG. 10. Comparison of the total reaction cross sections obtained by the Glauber (horizontal axis) and BS models (vertical axis). A solid-thin line indicates a $y=x$ line plotted to guide the eyes.

\section{E. Diffuseness of proton and neutron surfaces}

To extract detailed structure information of unstable nuclei, separation of proton and neutron diffuseness is important because the neutron diffuseness is expected to be more sensitive to the ground-state structure of neutron-rich isotopes as it is dominated by the neutron motion at the nuclear surface. As was done in Refs. [16,17], neutron distributions of stable nuclei can be extracted from the proton-nucleus elastic scattering cross section measurements using a known proton density distribution, but it is in general unknown for unstable nuclei. Here, we discuss the possibility of making use of the incident energy dependence of the $p n$ and $p p$ total cross sections as utilized in Refs. $[35,44]$. We extend that idea in order to extract both the proton and neutron surface diffuseness and radii.

We respectively assume the $2 \mathrm{pF}$ density distributions (6) for proton and neutron and determine these four parameters in such a way so as to reproduce the first peak positions and their differential elastic scattering cross sections at low and high incident energies. A realistic profile function [33], which differs for $p p$ and $p n$, is used for the Glauber calculation. Table I lists the extracted diffuseness parameters and rms radii for proton and neutron. Stable ${ }^{120} \mathrm{Sn},{ }^{208} \mathrm{~Pb}$ and neutronrich ${ }^{132} \mathrm{Sn}$ isotopes are chosen as the examples. We choose several sets of two incident energies among 200, 300, 550, and $800 \mathrm{MeV}$. For ${ }^{120} \mathrm{Sn}$ and ${ }^{208} \mathrm{~Pb}$, extracted diffuseness parameters are scattered although the rms radii are converged within $\sim 0.5 \%$. In such cases where the proton and neutron surfaces are located at almost the same position, the separation of the proton and neutron surface profiles might be difficult, whereas, in case of ${ }^{132} \mathrm{Sn}$, all extracted values are consistent with each other. This method can be used to extract the information on the proton and neutron surfaces from the proton-nucleus elastic scattering in the inverse kinematics, although the application of the method is limited only to such neutron(proton)-rich systems that the surfaces of the proton and neutron density distributions are well separated $\gtrsim 0.2 \mathrm{fm}$.

It should be noted the separation of the proton and neutron density distributions will be better by employing the cross sections at lower incident energies $\lesssim 200 \mathrm{MeV}$, where the $p n$ total cross section becomes much larger than that of the $p p$ one. As demonstrated in Ref. [35], the adopted Glauber model is reliable for a wide range of the incident energies, even at few tens of MeV. However, with lowering the incident energy, in-medium effects such as Pauli blocking and Fermi motion would be important and may modify the parameters of the free $N N$ profile function $[45,46]$. Implementing these effects will

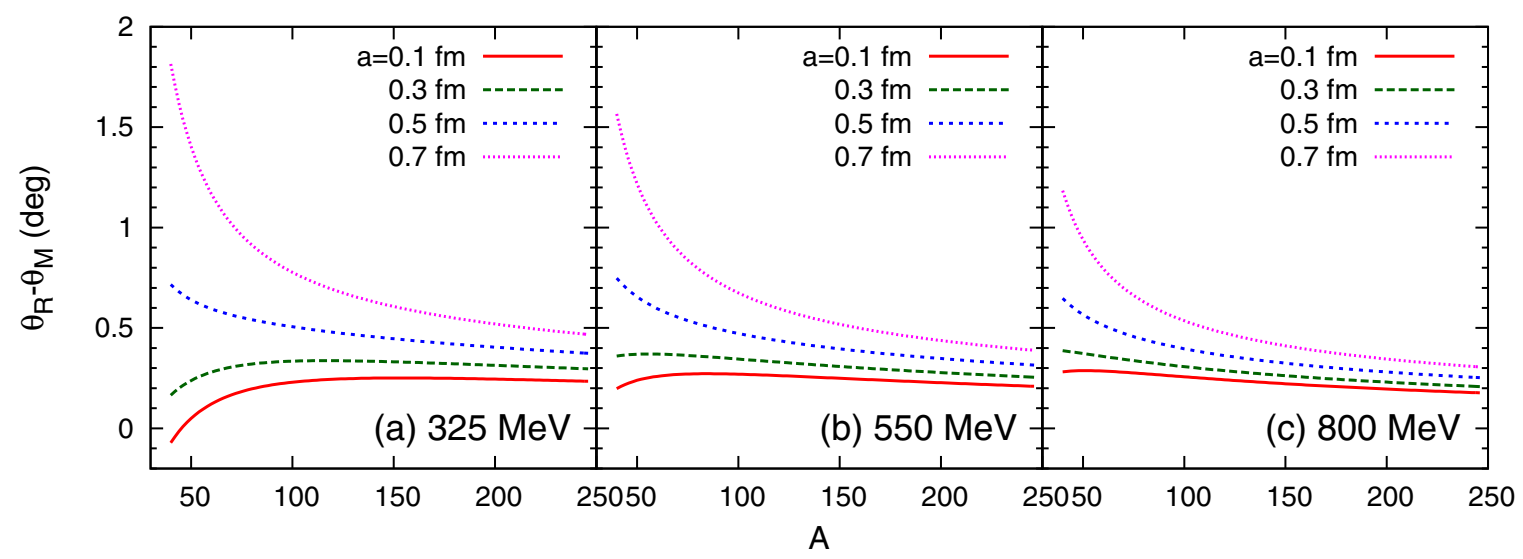

FIG. 11. Difference of the scattering angles of the first peak position obtained by the Glauber calculation and the BS estimate incident at (a) 325 , (b) 550 , and (c) $800 \mathrm{MeV}$. 
TABLE I. Diffuseness parameters and rms radii for matter $\left(r_{m}\right)$, neutron $\left(r_{n}\right)$, and proton $\left(r_{p}\right)$ extracted from the HF+BCS density distributions of ${ }^{120,132} \mathrm{Sn}$ and ${ }^{208} \mathrm{~Pb}$. at several choices of two incident energies among 200, 300, 550, and $800 \mathrm{MeV}\left(E_{L}<E_{H}\right)$. Units are given in $\mathrm{MeV}$ and fm for energy and length, respectively.

\begin{tabular}{lcccccc}
\hline \hline Nuclide & $\left(E_{L}, E_{H}\right)$ & $r_{m}$ & $r_{n}$ & $a_{n}$ & $r_{p}$ & $a_{p}$ \\
\hline${ }^{120} \mathrm{Sn}$ & $(200,300)$ & 4.691 & 4.725 & 0.455 & 4.645 & 0.619 \\
& $(200,550)$ & 4.686 & 4.720 & 0.506 & 4.639 & 0.507 \\
& $(200,800)$ & 4.685 & 4.724 & 0.470 & 4.629 & 0.525 \\
& $(300,550)$ & 4.683 & 4.708 & 0.543 & 4.648 & 0.455 \\
& $(300,800)$ & 4.683 & 4.713 & 0.543 & 4.640 & 0.448 \\
& $\mathrm{HF}+\mathrm{BCS}$ & 4.662 & 4.723 & & 4.576 & \\
${ }^{208} \mathrm{~Pb}$ & $(200,300)$ & 5.580 & 5.604 & 0.492 & 5.542 & 0.604 \\
& $(200,550)$ & 5.575 & 5.608 & 0.532 & 5.424 & 0.507 \\
& $(200,800)$ & 5.574 & 5.613 & 0.542 & 5.514 & 0.479 \\
& $(300,550)$ & 5.571 & 5.592 & 0.558 & 5.538 & 0.463 \\
& $(300,800)$ & 5.570 & 5.603 & 0.557 & 5.519 & 0.458 \\
& $\mathrm{HF}+\mathrm{BCS}$ & 5.551 & 5.617 & & 5.448 & \\
${ }^{132} \mathrm{Sn}$ & $(200,300)$ & 4.821 & 4.851 & 0.539 & 4.776 & 0.448 \\
& $(200,550)$ & 4.823 & 4.856 & 0.539 & 4.765 & 0.445 \\
& $(200,800)$ & 4.822 & 4.875 & 0.535 & 4.723 & 0.443 \\
& $(300,550)$ & 4.818 & 4.844 & 0.539 & 4.779 & 0.446 \\
& $(300,800)$ & 4.820 & 4.852 & 0.537 & 4.763 & 0.445 \\
& $\mathrm{HF}+\mathrm{BCS}$ & 4.802 & 4.890 & & 4.656 & \\
\hline \hline
\end{tabular}

be interesting for further improvement of the adopted Glauber model.

\section{CONCLUSION}

In order to see how much we can extract information on density profiles of unstable nuclei at around the nuclear surface, we have performed a numerical experiment using theoretically obtained elastic scattering differential cross sections of highenergy nucleon-nucleus scattering incident at a few to several hundreds of $\mathrm{MeV}$. The high-energy nucleon-nucleus collision is described by the Glauber model starting from the nucleonnucleon total cross sections.

We have demonstrated that the elastic scattering differential cross section at the first diffraction peak reflects the nuclear density profile at around the half-density radius. This can be understood naturally by extending the idea of the black sphere (BS) model offering the one-to-one correspondence between the nuclear radius and the diffraction peak. The deviation of the BS picture from the actual nucleon-nucleus scattering exhibits the role of the nuclear transparency due to the diffused nuclear surface. We have understood that the BS model is accurate but accompanied with typically $\sim 5 \%$ uncertainties in mediummass nuclei, at most $\sim 10 \%$ uncertainties in $\mathrm{Pb}$ isotopes.

Towards the application to studies of unstable nuclei, since experimental data of the elastic scattering differential cross sections at large scattering angles are hardly obtained, we restrict ourselves to have only two observables, the first peak position and its magnitude of the elastic scattering differential cross section. Assuming that the two-parameter Fermi $(2 \mathrm{pF})$ density distribution as a fitting density, we can uniquely determine these two parameters, from which the root-mean- square (rms) radius and nuclear diffuseness can be extracted. A systematic numerical experiment is performed using realistic density distributions obtained by a microscopic mean-field model. The accuracy of the extraction does not depend much on the incident energy. Though the simple $2 \mathrm{pF}$ form is assumed as an approximate density distribution, the rms radius can be determined within $\sim 1 \%$, and the extracted nuclear diffuseness is robust structure information that reflects interesting surface profiles on the exotic nuclei such nuclear deformation and shell evolution.

Since we only need the cross section at the first peak position, this method has a great advantage to apply to measurements in the inverse kinematics, in which the scattered particles are concentrated at the forward angles. The nuclear structure is actually reflected in the nuclear density profile at the surface, that is, the nuclear diffuseness. A systematic measurement along this direction is interesting to understand structure changes of unstable nuclei played by excess neutrons.

A prescription of separating the proton and neutron radii and diffuseness is also given by making use of the incident energy dependence of the proton-nucleus scattering. If one measures the elastic scattering differential cross sections at the first diffraction peak at low $(E \lesssim 300 \mathrm{MeV})$ and high $(E \gtrsim 500 \mathrm{MeV}$ ) incident energies, one can extract the surface diffuseness and the rms radii of proton and neutron separately. Though the method has the limitation that it can only be applied to a nucleus with large neutron-skin thickness, it will be useful to extract the structure information of neutron- and proton-rich unstable nuclei. We note, however, some simplifications of the model are made in this analysis. In order to obtain more precise information on the density profile, we need a further study to quantify the systematic error of this analysis with a more elaborated model, which includes many-fermion correlations as well as using a more flexible input density distribution.

We should point out here that the discussion extended in this paper must be applicable to the analyses of electron-nucleus elastic scattering differential cross sections, particularly for unstable nuclei, because the theoretical structure is quite similar to the proton scattering case [37], although the interaction is completely different. By focusing on the first peak angle and its magnitude of the elastic scattering differential cross section, one may have a chance to determine the radius of the proton density distribution and its diffuseness for an unstable nucleus simultaneously. We believe that this is very important, but leave it for our future studies [47].

\section{ACKNOWLEDGMENTS}

The authors thank K. Iida for valuable communications and $\mathrm{S}$. Ebata for sending us numerical data of density distributions obtained by the HF+BCS calculation. This work was in part supported by JSPS KAKENHI Grants No. 18K03635 and No. $18 \mathrm{H} 04569$.

\section{APPENDIX: TESTS OF AVERAGED $N N$ PROFILE FUNCTIONS}

In this Appendix, we test the validity of the average procedure of $p n$ and $p p$ total cross section made in the profile 

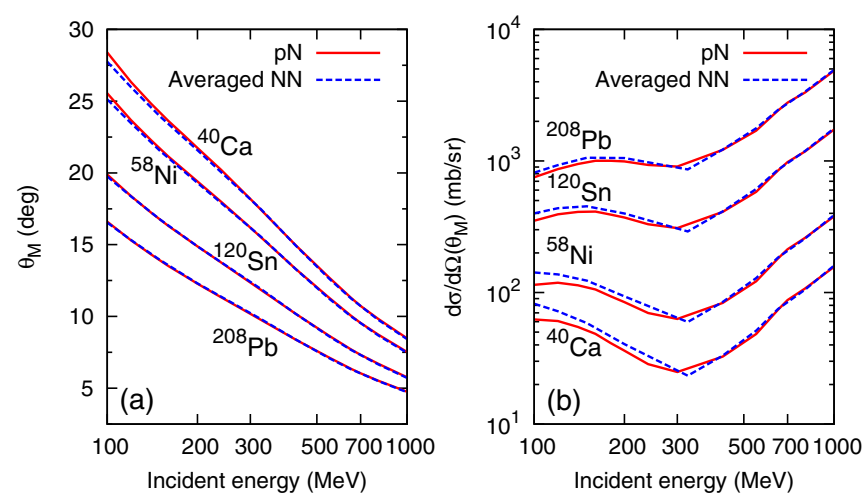

FIG. 12. (a) Scattering angles of the first peak position and (b) its magnitude of the elastic scattering differential cross section at the peak position with the $p N$ and averaged $N N$ profiles functions. The HF+BCS density distributions with the SkM* interaction $[35,39]$ are used. functions [32] for nucleon-nucleus systems employed in the analysis of this paper. We calculate the first peak positions $\theta_{M}$ and its magnitude of the elastic scattering differential cross section. The proton and neutron density distributions obtained by the HF+BCS method using the SkM* effective interaction [35,39] are employed. Figure 12 compares the results calculated with the $p N$ [33] and averaged $N N$ [32] profile functions at various incident energies. We find that the peak positions do not depend on the choice of the profile functions, while some differences are found in the cross sections at $\theta_{M}$ at incident energies lower than $\sim 300 \mathrm{MeV}$, where the difference of the $p n$ and $p p$ total cross section becomes significant. We can safely use the averaged $N N$ profile function for the scattering at $\gtrsim 300 \mathrm{MeV}$, the $p N$ profile functions should be used for quantitative discussions of the proton-nucleus scattering at the lower energies.
[1] H. de Vries, C. W. de Jager, and C. de Vries, At. Data Nucl. Data Tables 36, 495 (1987).

[2] I. Tanihata, H. Hamagaki, O. Hashimoto, Y. Shida, N. Yoshikawa, K. Sugimoto, O. Yamakawa, T. Kobayashi, and N. Takahashi, Phys. Rev. Lett. 55, 2676 (1985).

[3] T. Suzuki, H. Geissel, O. Bochkarev et al., Phys. Rev. Lett. 75, 3241 (1995).

[4] M. Takechi, T. Ohtsubo, T. Kuboki et al., Mod. Phys. Lett. A 25, 1878 (2010).

[5] K. Minomo, T. Sumi, M. Kimura, K. Ogata, Y. R. Shimizu, and M. Yahiro, Phys. Rev. C 84, 034602 (2011).

[6] K. Minomo, T. Sumi, M. Kimura, K. Ogata, Y. R. Shimizu, and M. Yahiro, Phys. Rev. Lett. 108, 052503 (2012).

[7] T. Sumi, K. Minomo, S. Tagami, M. Kimura, T. Matsumoto, K. Ogata, Y. R. Shimizu, and M. Yahiro, Phys. Rev. C 85, 064613 (2012).

[8] W. Horiuchi, T. Inakura, T. Nakatsukasa, and Y. Suzuki, Phys. Rev. C 86, 024614 (2012).

[9] S. Watanabe, K. Minomo, M. Shimada et al., Phys. Rev. C 89, 044610 (2014).

[10] M. Takechi, S. Suzuki, D. Nishimura et al., Phys. Rev. C 90, 061305(R) (2014).

[11] T. Inakura, T. Nakatsukasa, and K. Yabana, Phys. Rev. C 88, 051305(R) (2013).

[12] T. Inakura, W. Horiuchi, Y. Suzuki, and T. Nakatsukasa, Phys. Rev. C 89, 064316 (2014).

[13] S. Ebata, T. Nakatsukasa, and T. Inakura, Phys. Rev. C 90, 024303 (2014).

[14] W. Horiuchi, S. Hatakeyama, S. Ebata, and Y. Suzuki, Phys. Rev. C 96, 024605 (2017).

[15] H. Sakaguchi and J. Zenihiro, Prog. Part. Nucl. Phys. 97, 1 (2017), and references therein.

[16] S. Terashima, H. Sakaguchi, H. Takeda et al., Phys. Rev. C 77, 024317 (2008).

[17] J. Zenihiro, H. Sakaguchi, T. Murakami et al., Phys. Rev. C 82, 044611 (2010).

[18] G. Placzek and H. A. Bethe, Phys. Rev. 57, 1075 (1940).
[19] R. J. Glauber, Lectures in Theoretical Physics, edited by W. E. Brittin and L. G. Dunham (Interscience, New York, 1959), Vol. 1, p. 315.

[20] A. Kohama, K. Iida, and K. Oyamatsu, Phys. Rev. C 69, 064316 (2004).

[21] A. Kohama, K. Iida, and K. Oyamatsu, Phys. Rev. C 72, 024602 (2005).

[22] A. Kohama, K. Iida, and K. Oyamatsu, J. Phys. Soc. Jpn. 85, 094201 (2016).

[23] K. Iida, A. Kohama, and K. Oyamatsu, J. Phys. Soc. Jpn. 76, 044201 (2007).

[24] K. Varga, S. C. Pieper, Y. Suzuki, and R. B. Wiringa, Phys. Rev. C 66, 034611 (2002).

[25] T. Nagahisa and W. Horiuchi, Phys. Rev. C (to be published).

[26] B. Abu-Ibrahim, S. Iwasaki, W. Horiuchi, A. Kohama, and Y. Suzuki, J. Phys. Soc. Jpn. 78, 044201 (2009).

[27] R. H. Bassel and C. Wilkin, Phys. Rev. 174, 1179 (1968).

[28] S. Hatakeyama, S. Ebata, W. Horiuchi, and M. Kimura, J. Phys.: Conf. Ser. 569, 012050 (2014).

[29] S. Hatakeyama, S. Ebata, W. Horiuchi, and M. Kimura, JPS Conf. Proc. 6, 030096 (2015).

[30] Y. Suzuki, R. G. Lovas, K. Yabana, and K. Varga, Structure and Reactions of Light Exotic Nuclei (Taylor \& Francis, London, 2003).

[31] L. Ray, Phys. Rev. C 20, 1857 (1979).

[32] W. Horiuchi, Y. Suzuki, B. Abu-Ibrahim, and A. Kohama, Phys. Rev. C 75, 044607 (2007).

[33] B. Abu-Ibrahim, W. Horiuchi, A. Kohama, and Y. Suzuki, Phys. Rev. C 77, 034607 (2008); 80, 029903 (2009); 81, 019901 (2010).

[34] G. D. Alkhazov, S. L. Belostotsky, and A. A. Vorobyov, Phys. Rep. 42, 89 (1978).

[35] W. Horiuchi, S. Hatakeyama, S. Ebata, and Y. Suzuki, Phys. Rev. C 93, 044611 (2016).

[36] A. Bohr and B. R. Mottelson, Nuclear Structure, Vols. I \& II (W. A. Benjamin, New York, 1975).

[37] R. D. Amado, J. P. Dedonder, and F. Lenz, Phys. Rev. C 21, 647 (1980). 
[38] S. Ebata, T. Nakatsukasa, T. Inakura, K. Yoshida, Y. Hashimoto, and K. Yabana, Phys. Rev. C 82, 034306 (2010).

[39] W. Horiuchi, S. Ebata, and K. Iida, Phys. Rev. C 96, 035804 (2017).

[40] S. Ebata, Y. Hirabayashi, S. Hatakeyama, and W. Horiuchi (unpublished).

[41] J. Bartel, P. Quentin, M. Brack, C. Guet, and H. Håkansson, Nucl. Phys. A 386, 79 (1982).

[42] S. Ebata and T. Nakatsukasa, Phys. Scr. 92, 064005 (2017).
[43] W. Horiuchi, T. Inakura, T. Nakatsukasa, and Y. Suzuki, JPS Conf. Proc. 6, 030079 (2015).

[44] W. Horiuchi, Y. Suzuki, and T. Inakura, Phys. Rev. C 89, 011601(R) (2014).

[45] M. S. Hussein, R. A. Rego, and C. A. Bertulani, Phys. Rep. 201, 279 (1991).

[46] M. Takechi, M. Fukuda, M. Mihara et al., Phys. Rev. C 79, 061601(R) (2009).

[47] K. Tsukada, A. Enokizono, T. Ohnishi et al., Phys. Rev. Lett. 118, 262501 (2017). 